\title{
A Cost-Based Model of Seasonal Production, with Application to Milk Policy
}

\author{
David A. Hennessy and Jutta Roosen
}

Working Paper 03-WP 323

January 2003

\author{
Center for Agricultural and Rural Development \\ lowa State University \\ Ames, lowa 50011-1070 \\ www.card.iastate.edu
}

David A. Hennessy is a professor in the Department of Economics and the Center for Agricultural and Rural Development, lowa State University. Jutta Roosen is an assistant professor, Faculté de Sciences Agronomique, University Catholique de Louvain, Louvain-la-Neuve, Belgium.

This journal paper of the lowa Agriculture and Home Economics Experiment Station, Ames, lowa, Project No. 3463, was supported by Hatch Act and State of lowa funds.

This publication is available online on the CARD website: www.card.iastate.edu. Permission is granted to reproduce this information with appropriate attribution to the authors and the Center for Agricultural and Rural Development, lowa State University, Ames, lowa 50011-1070.

For questions or comments about the contents of this paper, please contact David Hennessy, 578C Heady Hall, lowa State University, Ames, IA 50011-1070; Ph: 515-294-6171; Fax: 515-2946336; E-mail: hennessy@iastate.edu.

lowa State University does not discriminate on the basis of race, color, age, religion, national origin, sexual orientation, sex, marital status, disability, or status as a U.S. Vietnam Era Veteran. Any persons having inquiries concerning this may contact the Director of Equal Opportunity and Diversity, 1350 Beardshear Hall, 515-294-7612. 


\begin{abstract}
Milk production is seasonal in many European countries. While quantity seasonality poses capacity management problems for dairy processors, a European Union policy goal is to reduce price seasonality. After developing a model of endogenous seasonality, we study the effects of three E.U. policies on production decisions. These are private storage subsidies, production removals, and production quotas. When cost functions are seasonal in a specified way, then arbitrage opportunities interact with storage subsidies to reduce both price and consumption seasonality. But production seasonality likely increases because storage subsidies promote temporal market integration. Conditions are identified under which product market interventions increase quantity seasonality.
\end{abstract}

Keywords: efficiency, market intervention, quota, stabilization, storage subsidies. 


\title{
A COST-BASED MODEL OF SEASONAL PRODUCTION, WITH APPLICATION TO MILK POLICY
}

\author{
1. Introduction \\ Milk production is characterized by a high degree of seasonality in some countries of \\ the European Union. This is a striking feature of production patterns in Ireland where milk \\ producers rely mostly on summer-grazing, spring-calving systems, and 85 percent of \\ manufacturing milk is produced from March through October (Crosse, O'Brien, and Ryan, \\ 2000). This strong seasonality has important implications for the efficiency of bovine \\ agriculture. While the production of milk is highly seasonal, demand for dairy products is \\ quite stable throughout the year. Highly seasonal patterns generate a need to build suffi- \\ cient (peak-load) capacities for transporting and, as milk is a perishable product, processing \\ peak production. This leads to idle capacity during months of low supply. \\ Milk from spring-calving herds pose an additional problem in the processability of \\ milk. Late-lactation milk is in general of lower quality, as measured in somatic cell \\ counts, total bacterial counts, and free fatty acids. Crosse, O'Brien, and Ryan (2000) \\ suggest that a consequence of seasonality is that dairy processing will occur only over \\ limited intervals during the year. With non-convexities in processing cost structures, \\ processors facing seasonal milk inflows may find it unprofitable to engage extensively in \\ high-value-added processing of the better-quality milk they do receive. Nor are the \\ implications of dairying seasonality confined to that sector. Seasonal patterns in calving \\ contribute to seasonal patterns in beef production, and here again the costs may be large. \\ Apart from direct capacity management issues, increased line speed in animal slaughter- \\ ing facilities may contribute to an increase in carcass pathogen contaminations (Bell, \\ 1997; Sheridan, 1998). ${ }^{1}$ \\ While it technically may be feasible to adjust the natural milk supply patterns, any at- \\ tempt to do so through policy interventions must satisfy biotechnological constraints. Caine \\ and Stonehouse (1983) have proposed two different policy measures to adjust seasonal
}


milk supply in Ontario, Canada. One is a quarterly quota system and the other is a seasonal subsidy payment to encourage milk production during winter. The use of pricing schemes to adjust the seasonality of milk production is, however, not as simple as it may seem at first glance. While the low volume of winter milk suggests that it may come at a premium, the poor quality of late-lactation (thus, mainly winter) milk has encouraged the use of payment schemes that reward summer production. ${ }^{2}$ Any pricing scheme designed to promote quality and also alter seasonal production must recognize that the underlying biotechnology imposes interactions between season and quality.

When deciding on a specific calving system, and hence on its milk production pattern, farmers have to account for various economic parameters. These include the abundance of low-cost feed during summer months, the seasonality of milk prices, and how quality affects prices. But the policy environment is also important, and in this paper we are concerned with policies that affect seasonality. In particular, we look at two aspects of E.U. milk policies. While commercial milk prices are set in private markets, the European Union has available a number of policy instruments that can alter the level and seasonal pattern of prices. One instrument is the subsidization of private storage activities in milk product markets. A second is intervention to permanently remove product from the marketplace. We also are concerned with how a third policy, the maintenance of milk marketing quotas, might modify the effects of storage subsidy and removal policies on seasonality.

With the intent to stabilize markets, the E.U. Common Agricultural Policy (CAP) seeks to reduce price seasonality through subsidies that encourage the private purchase and storage of product at times of high production for sale at times of low production. In a two-season model, we provide precise conditions that support the intuition that seasonal cost advantages lead to seasonal production patterns in markets where product is storable. And we establish that, whether or not a quota regime is in place, a storage subsidy dampens price seasonality by amplifying production seasonality. We also will show that the overall incentive to produce may rise or fall with a storage subsidy.

In studying interventions to support market prices by permanently removing product from the market when the price is low, we also consider environments in which quotas are and are not in effect. Our two-season model is extended to establish the rather nonintuitive 
result that, absent a quota regime, such product removal interventions could dampen production seasonality under plausible assumptions about the production technology. When a quota regime is in place, the interventions have no effect on production seasonality.

The paper is structured as follows. After a brief review of seasonality in milk production and of relevant E.U. policy, we present formal treatments of a policy to subsidize private storage and a policy of permanent product removal in the trough price season. In each case, these treatments are first presented absent a quota regime, and then with the imposition of a quota regime. The paper concludes with a brief discussion of policy issues.

\section{Background}

Commercial dairy production in northwestern Europe has long been characterized by seasonality. This is due mainly to managerial decisions to spring calve in order to take advantage of cheap summer forage. While still a prominent feature of milk production in many locations, quantity seasonality has become less pronounced in recent years as new forage conservation methods have become available. The use of artificial insemination and improved housing conditions for dairy cows also may have contributed to reduced seasonality. ${ }^{3}$

Figure 1 compares two snapshots (the years 1975 and 2000) of the milk production pattern in Ireland, where approximately 5 percent of E.U. milk is produced. It can be seen that, when normalized to control for the overall increase in dairy production, the strongly seasonal pattern has declined somewhat. The standard deviation of monthly production shares decreased from 0.052 in 1975 to 0.044 in 2000. However, in many other European countries the seasonality pattern developed quite differently. For instance, the standard deviation of monthly production shares decreased from 0.011 to 0.006 in the United Kingdom and from 0.015 to 0.009 in France between 1973 and 1997. Figure 2 confirms that Irish milk production, in contrast to other countries, has remained highly seasonal, with a 7 to 1 ratio of peak monthly production (May) to trough monthly production (January). ${ }^{4}$

The persistence of strongly seasonal production in Ireland may have a variety of contributing factors. First, compared with other countries in northern Europe, Ireland has a low population density and so has low local demand for liquid milk. In the path of the Gulf Stream and the accompanying rain clouds, Ireland is endowed with a climate that is 


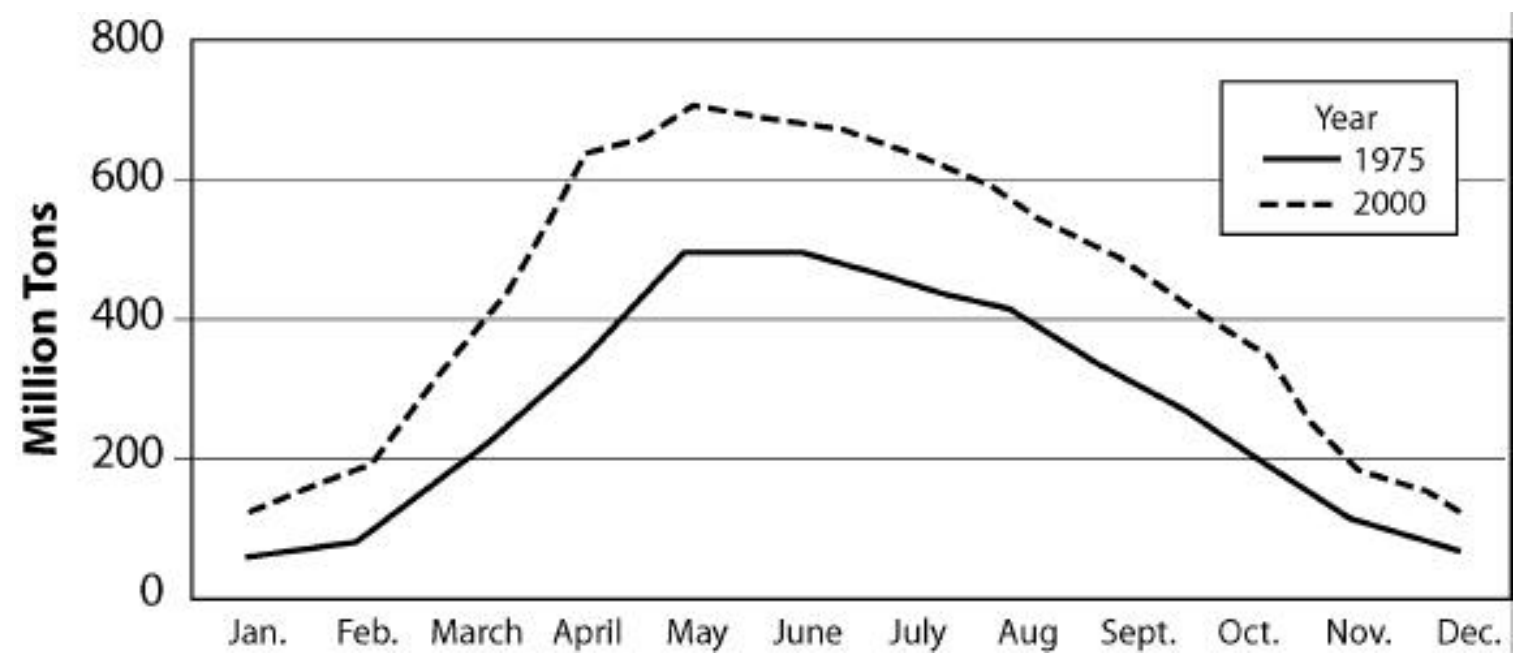

FIGURE 1. Changes in the seasonality of milk production in Ireland in million tons of milk intake by creameries and pasteurizers per month

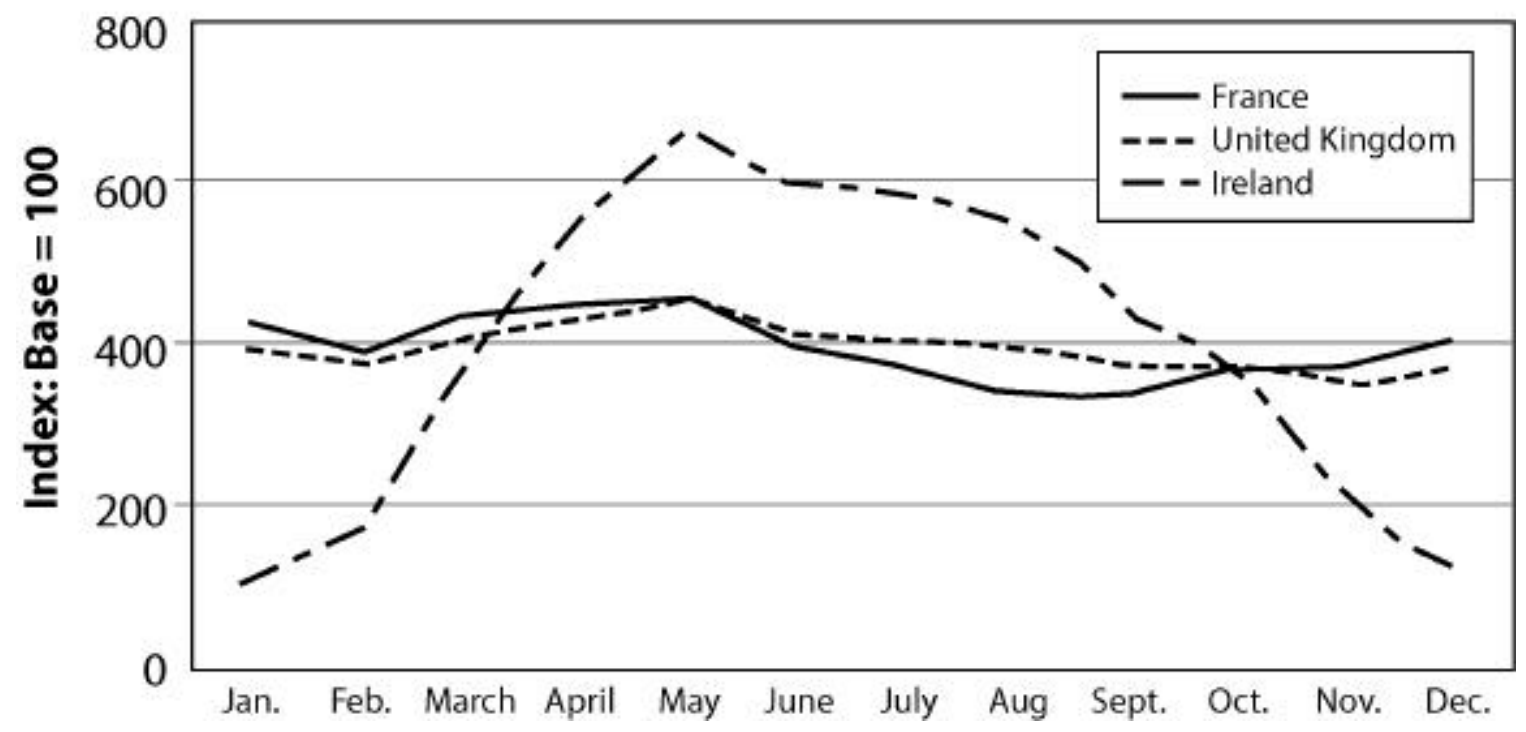

FIGURE 2. Seasonality of milk production relative to annual production in France, Ireland, and the United Kingdom, 1999

favorable to grass growth between April and October. At lower elevations in East Munster and South Leinster, this climate meets soils that are well suited for grass growth. As a result, dairying is a popular enterprise, and almost all milk production is destined for processing. In addition, it is relatively easy to store, at a cost, many processed dairy products. With milk for processing, farmers are relatively free to make the best use of cheap in 
situ summer grazing. And so, the overwhelming majority of Irish milk herds are springcalving herds. Irish farmers do not incur much of a price penalty for producing in the summer. As can be seen from Figure 3, while farm-level prices are counterseasonal relative to quantity seasonality, the amplitude of price seasonality has been less than one-fifth that of quantity seasonality in recent years. Over the latter part of the 1990s, Irish price seasonality broadly has been comparable with patterns in France and the United Kingdom.

Also, the persistence of seasonality in Irish milk production patterns may be explained by the conjoin of a fact and a conjecture, both of which were alluded to earlier. The fact is that milk quality declines as the parturition date recedes. The conjecture is that endogenous quantity seasonality in production may be, in part, determined by the presence of processing technology nonconvexities. It might be speculated that multiple equilibria exist, whereby processors that receive a relatively smooth supply of milk with relatively constant shares of higher-quality milk over the year are in a better position to market higher-value products. These firms then may have stronger incentives to encourage further deseasonalization of milk production to develop their market for the higher-valued products. Other firms may conclude that it is futile to compete with entrenched market leaders possessed of an endowed advantage in the nature of raw milk supplies.

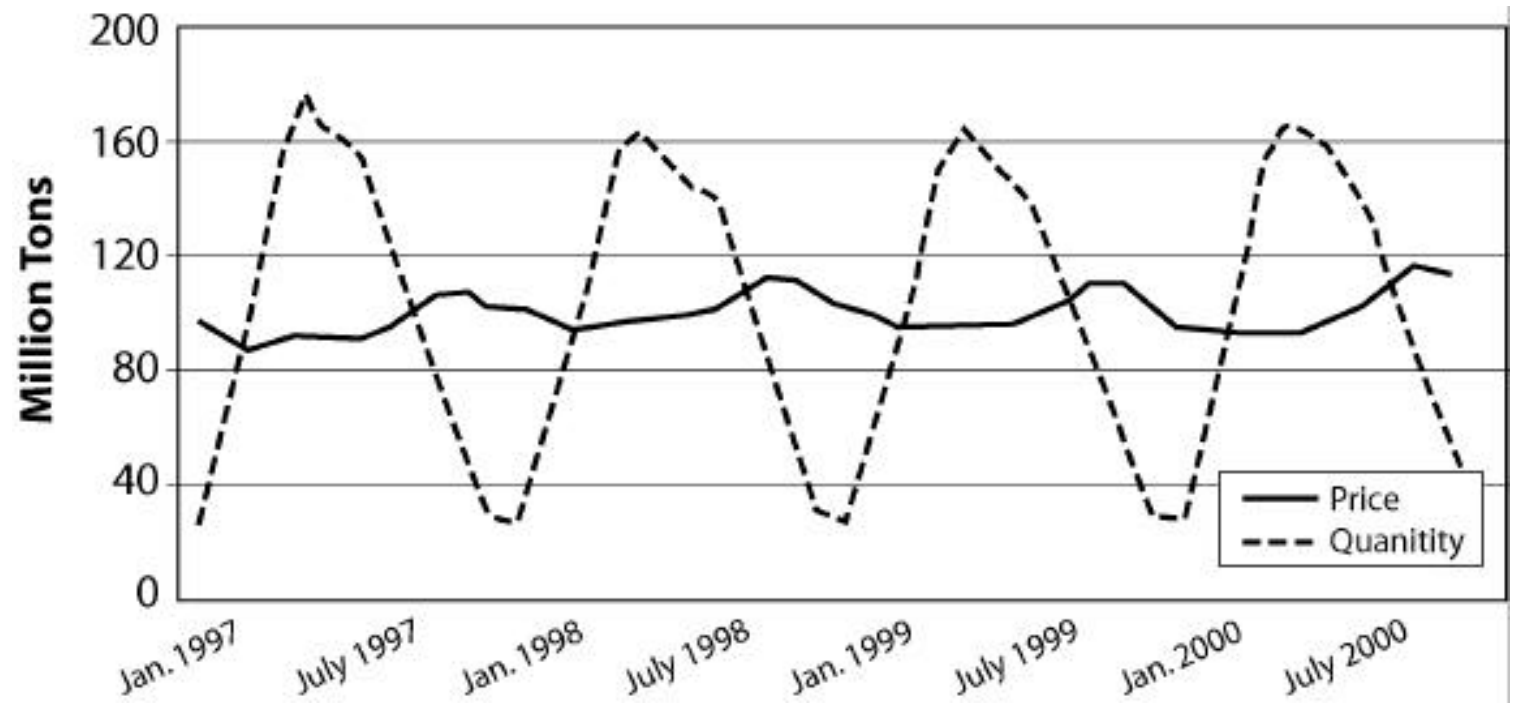

FIGURE 3. Irish milk seasonality, 1997-2000: quantity and price 
In this paper we investigate a third possibility that may influence the persistence of quantity seasonality in milk production: that E.U. policy may be a contributing factor. Before formalizing our argument, we will outline the nature of the policies at issue. ${ }^{5} \mathrm{CAP}$ subsidies on private storage are supported for meat and dairy products. As of May 2002, regulations $2771 / 99,1362 / 87$, and 2659/94 stipulate the respective conditions for butter, skim milk powder (SMP), and cheeses. In the case of butter and cream, the storage period is March 15 through August 15, while the SMP scheme also is most likely to be triggered during peak seasonal production. The subsidies take the form of a fixed amount per ton sequestered, a daily rate per ton, and an interest rate subsidy.

The European Union also is empowered to intervene directly in butter and SMP markets. For butter, intervention is triggered when market prices fall below 90 percent of the reference intervention price, and intervention purchases are suspended when market prices exceed 92 percent of the reference price. Low market prices also trigger intervention in SMP markets, but such interventions can only occur between March 1 and August $31{ }^{6}$ Next, we develop a two-season model, with high-cost and low-cost seasons, that teases out the effects of storage subsidies and milk product market interventions on seasonality in production and consumption.

\section{Storage Subsidies and Product Market Interventions}

\subsection{Model}

For the sake of simplicity we hold that the milk production year can be divided into two seasons, each of equal duration. Respective output levels $q_{a}=\hat{q}+\phi$ and $q_{b}=\hat{q}-\phi$ are produced in seasons A and B, where the levels of $\hat{q}$ and $\phi$ shall be endogenous to our model. ${ }^{7}$ We label $\phi$ the production seasonality index because it can be viewed as an index of deterministic variability in production. And $\hat{q}$, being the nonseasonal component of production per season, is labeled the production index. Without compromising our argument, the intertemporal interest rate is assumed to have zero value. The market is perfectly competitive, and there are three categories of agents.

In the first category we have the producers and, in the aggregate, their annual cost function for inputs other than those where unit costs vary by season. Typically, feed, fertilizer, labor, and some parts of utility bills can be represented by the twice continu- 
ously differentiable and strictly convex function $R(\hat{q}+\phi, \hat{q}-\phi)$ which is defined on $\mathbb{R}_{+}^{2}{ }^{8}$ This function is held to be permutation symmetric in the sense that $R\left(\mathrm{q}_{\mathrm{a}}=q_{1}, q_{b}=q_{2}\right)=R\left(q_{a}=q_{2}, q_{b}=q_{1}\right) \forall\left(q_{1}, q_{2}\right) \in \mathbb{R}_{+}^{2}$ where $q_{1}$ and $q_{2}$ are evaluations of the two arguments. Because we have factored out the seasonal cost component, we believe that symmetry and convexity are quite intuitive assumptions. Together, the assumptions imply that unbalanced seasonal production carries with it a cost penalty, but the penalty is independent of whether the larger amount is produced in season A or season B. For management costs and for the maintenance costs of such things as providing water, electricity, bulk tanks, and an adequate parlour, this seems reasonable. ${ }^{9}$

Unit seasonal costs are given, again in deviations form, by $m_{a}=\frac{1}{2}(m-\rho)$ and $m_{b}=$ $\frac{1}{2}(m+\rho)$ where $\rho \geq 0$ and $m-\rho>0$, so that season A may be thought of as the (exogenously determined) low-cost summer grazing season. While $m$, which is a nonseasonal marginal cost, might be subsumed in $R\left(q_{1}, q_{2}\right)$, we will use it as a parameterization of nonseasonal marginal costs. Thus, aggregated over all producers, total on-farm production costs are given by the expression

$$
R(\hat{q}+\phi, \hat{q}-\phi)+(\hat{q}+\phi) m_{a}+(\hat{q}-\phi) m_{b}=R(\hat{q}+\phi, \hat{q}-\phi)+m \hat{q}-\rho \phi .
$$

Here we may think of $\rho \phi$ as the cost savings that are gleaned from managerial adjustments of $\hat{q}$ and $\phi$ to take best advantage of $\rho$.

The next category of agents is the processors. In the aggregate, their annual cost function can be represented by $D(\hat{q}+\phi, \hat{q}-\phi)$. This, too, is held to be twice continuously differentiable, symmetric, and strictly convex. In order to economize on expression lengths at a later juncture, we write $C(\hat{q}+\phi, \hat{q}-\phi)=D(\hat{q}+\phi, \hat{q}-\phi)+R(\hat{q}+\phi, \hat{q}-\phi)$, and note that it, too, is symmetric and convex.

ASSUMPTION 1. Cost function $C\left(q_{a}, q_{b}\right)$ is symmetric and strictly convex.

Finally, in the third category, we have the consumers. Our concern is mainly with production seasonality, for it is there that most of the seasonality in animal agriculture arises. ${ }^{10}$ In our quest to understand the interactions between seasonality and policy, we 
assume temporal separability and season invariant demand. In addition, we will often assume a linear inverse demand equation, $P(q)=\alpha_{0}-\alpha_{1} q$ where $\alpha_{0}>0, \alpha_{1}>0$, and $P(q)>0$ for all quantities of interest.

ASSUMPTION 2. The demand function is temporally separable, and season invariant.

ASSUMPTION $2^{\prime}$. The demand function is linear, temporally separable, and season invariant.

It bears emphasizing that seasonality does not originate on the demand side, where season invariant preferences underpin the season invariant inverse demand function given by the strictly decreasing function $P(q)$. While, as we will show, consumers may behave in a seasonal manner, this is an equilibrium response to production-side cost seasonality.

It is important to note that there is no uncertainty in our model, and our interest is only in an analysis of stationary equilibrium. Thus, while intertemporal product transfers may occur, they will be confined within some replicating year-long interval. If we identify consumption in season $V$ as $q_{v}^{c}$, then a stationary equilibrium requires that $q_{a}^{c}+q_{b}^{c}=\hat{q}+\phi+\hat{q}-\phi=2 \hat{q}$. In order to provide a convenient interpretation of the results that we will shortly establish, we write consumptions in deviation form, with $q_{a}^{c}=\hat{q}+\delta$ and $q_{b}^{c}=\hat{q}-\delta$. As well, $\delta$ may be interpreted as an endogenous index of seasonality in consumption. The season A difference between production and consumption, i.e., $\phi-\delta$, represents the amount of product transferred over seasons through storage. Finally, let the unit cost of storage be $s>0 .^{11}$

To review, note that we have developed and parameterized three concepts of seasonality. Two are endogenous: production seasonality as given by index $\phi$ and consumption seasonality as given by index $\delta$. The third, exogenous cost seasonality as represented by index $\rho$, is the technology parameter that motivates seasonal patterns in behavior.

Subject to one qualification, we can now write the expression for aggregate profit on the supply side, i.e., profit summed over all producers and all processors and over both periods, as 


$$
\begin{aligned}
\Pi[\delta, \phi, \hat{q}] & =P(\hat{q}-\delta)(\hat{q}-\delta)+P(\hat{q}+\delta)(\hat{q}+\delta)-(\phi-\delta) s \\
& -C(\hat{q}+\phi, \hat{q}-\phi)-m \hat{q}+\rho \phi .
\end{aligned}
$$

System convexity is assured by the strict convexity of $C(\hat{q}+\phi, \hat{q}-\phi)$. The qualification is that storage costs actually amount to $|\phi-\delta| s$, and we have not yet established that $\phi \geq \delta$. We will shortly provide conditions under which this is true. But before we do so, we take the liberty of assuming it so as to describe optimal behavior.

Notice that payments to farmers are transfer payments that net out in expression (1). Further, suppose that the socially optimal signals are sent from processors to producers. Then we may invoke the first fundamental welfare theorem (Mas-Colell, Whinston, and Green, 1995) because we know that competitive markets will then support the optimized choices of arguments $(\delta, \phi, \hat{q})$. And so, for the general demand structure given in Assumption 2, we have the price-taking optimality conditions:

(a) $\delta: P(\hat{q}-\delta)-P(\hat{q}+\delta)-s=0$,

(b) $\phi:-s-C_{1}(\hat{q}+\phi, \hat{q}-\phi)+C_{2}(\hat{q}+\phi, \hat{q}-\phi)+\rho=0$,

(c) $\hat{q}: P(\hat{q}-\delta)+P(\hat{q}+\delta)-C_{1}(\hat{q}+\phi, \hat{q}-\phi)-C_{2}(\hat{q}+\phi, \hat{q}-\phi)-m=0$,

where $C_{i}\left(q_{a}, q_{b}\right)$ is the differential with respect to the $i$ th argument. ${ }^{12}$ We have not presented the complementary slackness conditions because it is assumed that the solution is interior. From the law of demand, (2a) implies that $\delta>0$ for all $s>0$.

Condition (2a), the arbitrage criterion, is just the assertion that arbitrage opportunities do not exist, i.e., if it pays to store, then storage will occur until the price differential eliminates the profits from putting an extra unit into storage. Note in particular that if $s=$ 0 then $\delta=0$. Condition $(2 b)$, the allocation criterion, represents the exhaustion of cost efficiencies associated with transferring production from the high-cost period to the lowcost period. Condition $(2 c)$, the production criterion, represents the equation of summed marginal revenues and summed marginal costs due to a ray expansion of output, i.e., change $q_{a}$ and $q_{b}$ by the same small amount.

At this point, we invoke a result from the theory of majorization (Marshall and Olkin, 1979; Chambers and Quiggin, 1997 and 2000). For a permutation symmetric, 
convex, continuously differentiable function, $g(\vec{z}): I^{n} \rightarrow \mathbb{R}$, where $I^{n}$ is the convex interval product domain of relevance, the Ostrowski condition asserts that ${ }^{13}$

$$
\left(z_{i}-z_{j}\right)\left[g_{i}(\vec{z})-g_{j}(\vec{z})\right] \geq 0 \forall \vec{z}, \forall i, j \in(1, \ldots, n)
$$

is true. This inequality is of interest because we may employ it to rewrite $(2 b)$ above as

$$
(\rho-s) \phi=\left[C_{1}(\hat{q}+\phi, \hat{q}-\phi)-C_{2}(\hat{q}+\phi, \hat{q}-\phi)\right] \phi \geq 0
$$

Having made this observation, we can show the following.

RESULT 1. Under Assumption 1, if $\rho>s>0$ and $s$ is sufficiently small, then $\phi \geq \delta>0$. Therefore, objective function (1) is well posed.

All proofs are provided in the Appendix. ${ }^{14}$ It has already been assumed that there is cost seasonality, $\rho>0$. Result 1 demonstrates that storage from the low-cost season to the high-cost season will occur when arbitrage profits are possible, i.e., when the stronger inequality $\rho>s$ pertains. It merits observation that cost seasonality induces endogenous consumption seasonality, but consumption seasonality is tempered by the extent of the storage infrastructure.

Note that system (2) is possessed of some peculiarities that can be exploited in the analysis to follow. In particular, only endogenous variables $\hat{q}$ and $\delta$ arise in (2a) while only variables $\hat{q}$ and $\phi$ arise in $(2 b)$. Upon specializing to Assumption 2', linearity in demand generates the following system:

$$
\begin{aligned}
& \left(a^{\prime}\right) \delta 2 \alpha_{1} \delta=s, \\
& \left(b^{\prime}\right) \phi: \quad-C_{1}(\hat{q}+\phi, \hat{q}-\phi)+C_{2}(\hat{q}+\phi, \hat{q}-\phi)=s-\rho, \\
& \left(c^{\prime}\right) \hat{q}: 2 \alpha_{0}-2 \alpha_{1} \hat{q}-C_{1}(\hat{q}+\phi, \hat{q}-\phi)-C_{2}(\hat{q}+\phi, \hat{q}-\phi)=m .
\end{aligned}
$$

Linear demand is helpful because system (2') has become block separable, where $\left(2^{\prime} a^{\prime}\right)$ determines the value of $\delta$ and the other two equations jointly determine the values of $\hat{q}$ and $\phi$. 


\subsection{Storage Subsidies and Seasonality}

\subsubsection{No Quota}

Focusing on block $\left(2^{\prime} b^{\prime}\right)-\left(2^{\prime} c^{\prime}\right)$, storage costs arise only in $\left(2^{\prime} b^{\prime}\right)$. And the seasonality parameter enters in exactly the same manner so that, from the perspective of equilibrium determination, $s$ and - $\rho$ are indistinguishable. Comparative statics on the system immediately yield the following, which will be used as reference points for later deductions.

RESULT 2. Under assumptions 1 (i.e., symmetric, convex cost) and 2' (i.e., linear demand):

a. $d \delta / d s \geq 0, d[P(\hat{q}-\delta)-P(\hat{q}+\delta)] / d s \geq 0, d \delta / d m=0$ and $d[P(\hat{q}-\delta)-P(\hat{q}+\delta)] d m=0$. That is, both consumption seasonality and the peak-trough price difference are decreasing in a storage subsidy. They are unaffected by either a change in the level of cost seasonality or a change in the level of nonseasonal costs.

b. $d \phi / d s \leq 0$ and $d \phi / d \rho \geq 0$. That is, production seasonality increases under a storage subsidy or an increase in cost seasonality.

c. $d \hat{q} / d m \leq 0$. That is, production decreases with an increase in nonseasonal costs.

d. $d \hat{q} / d \rho=-d \phi / d m$. That is, the effect of a small increase in seasonal costs on nonseasonal production equals the effect of a small decrease in nonseasonal costs on production seasonality.

As these deductions are largely in accord with intuition, we will not dwell upon them. Notice, however, that we have related little about the effects of cost seasonality or a storage subsidy on the overall level of production, $\hat{q}$. Part $(d)$, which may be interpreted as a duality-type reciprocity result on the moments of milk supply, does impart some information. But no regularity assumptions made thus far allow us to sign either side of the equation. ${ }^{15}$ In fact, the effects can go either way. Suppose that the nonseasonal production technology is symmetric, being of the form $C^{*}\left(q_{a}, q_{b}\right)=\hat{C}\left(q_{a}\right)+\hat{C}\left(q_{b}\right)+\gamma q_{a} q_{b}, \gamma \in \mathbb{R}$. From work underlying Result 2, the following transpires. 
RESULT 3. Under assumptions 1 and $2^{\prime}$, let $C\left(q_{a}, q_{b}\right)$ have the form $C^{*}\left(q_{a}, q_{b}\right)$. Then:

a. $\quad d \hat{q} / d s \geq(\leq) 0$ whenever $\hat{C}_{111}(q) \geq(\leq) 0 \forall q \geq 0$. That is, the level of production increases (decreases) with either a storage subsidy or an increase in cost seasonality whenever $\hat{C}_{111}(q) \leq(\geq) 0 \forall q \geq 0$.

b. $\quad d \phi / d m \geq(\leq) 0$ whenever $\hat{C}_{111}(q) \leq(\geq) 0 \forall q \geq 0$. That is, the level of production seasonality, $\phi$, increases (decreases) with an increase in nonseasonal costs whenever marginal cost is convex (concave).

c. $\quad d P(\hat{q}-\delta) / d s \geq 0$ if $\hat{C}_{111}(q) \leq 0 \forall q \geq 0$, while $d P(\hat{q}+\delta y d s \leq 0$ if $\hat{C}_{111}(q) \geq 0 \forall q \geq 0$. That is, the peak price decreases (trough price increases) with a storage subsidy if marginal cost is concave (convex).

d. $\quad d P(\hat{q}-\delta) / d \rho \geq(\leq) 0$ whenever $\hat{C}_{111}(q) \geq(\leq) 0 \forall q \geq 0$ and $d P(\hat{q}+\delta) / d \rho \geq(\leq) 0$ whenever $\hat{C}_{111}(q) \geq(\leq) 0 \forall q \geq 0$. That is, both peak and trough prices increase (decrease) with an increase in cost seasonality whenever marginal cost is concave (convex).

Figure 4 illustrates the case where $\hat{C}_{111}(q) \geq 0 \forall q \geq 0$. The figure stresses three production points; $q, q+2 t$, and $q+4 t$, where $t>0$. The midpoints of the segments $(q, q+$ $2 t)$ and $(q+2 t, q+4 t)$ are also identified on the horizontal axis. For each midpoint, the vertical gap between the segment evaluation and the cost function evaluation is quantified on the vertical axis. This vertical gap reflects the increase in cost due to a two-point spread in production away from the segment midpoint. For example, the smaller vertical gap reflects the increase in nonseasonal costs associated with producing $q+2 t$ in season A and $q$ in season $\mathrm{B}$ rather than $q+t$ in each season. The claim we make is that condition $\hat{C}_{111}(q) \geq 0 \forall q \geq 0$ implies that, upon taking the limit as $t \rightarrow 0$, the vertical gap is larger on segment $(q+2 t, q+4 t)$ than on segment $(q, q+2 t)$. To demonstrate, we can write

$$
\Phi(t)=[\hat{C}(q+4 t)+\hat{C}(q+2 t)-2 \hat{C}(q+3 t)]-[\hat{C}(q+2 t)+\hat{C}(q)-2 \hat{C}(q+t)] .
$$




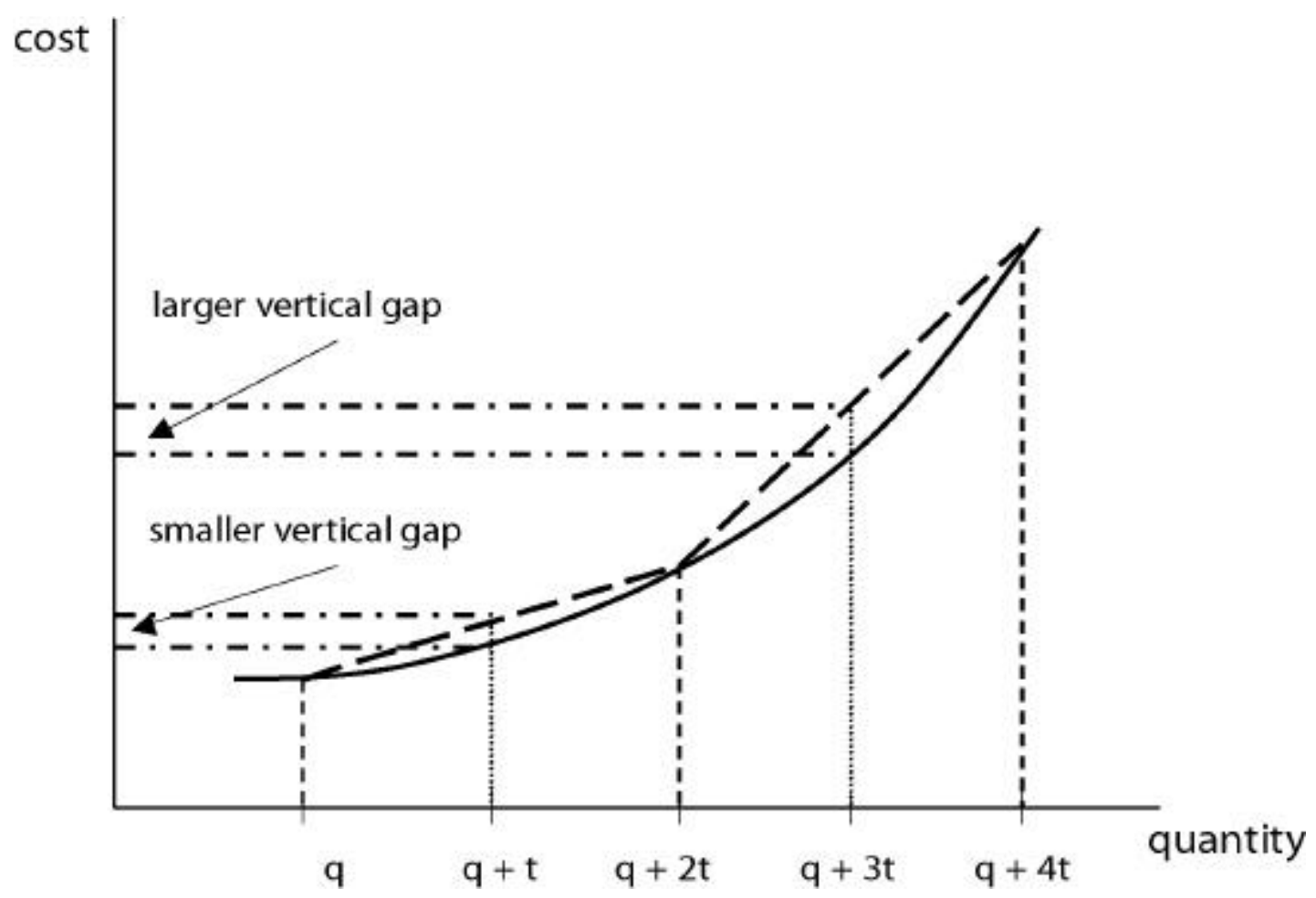

FIGURE 4. Characterizing a function with convex marginal cost

Upon differentiating and evaluating at $t=0$, we have $\Phi_{1}(t=0)=0, \Phi_{11}(t=0)=0$, and $\Phi_{111}(t=0)=12 \hat{C}_{111}(t=0)$. The intuition that Figure 4 provides is that variability in production is more costly at higher levels of output than at lower levels of output.

Intuition for part $(a)$ in Result 3 can be obtained by observing that a storage subsidy allows a more complete integration of the two markets. We saw, in Result 2(b), that a storage subsidy elevates temporal variation in production. Production decisions are made at the margin. If marginal cost is convex, then "expected" marginal cost increases with the advent of a storage subsidy. In this case, the incentive to produce is depressed. And that is precisely the economic content of Result 3(a). Production seasonality and the level of production are complements if marginal cost, suitably separated, is concave. Then a subsidy on, say, winter housing or winter fodder would reduce both production seasonality and the scale of production. 
Borrowing terminology from Kimball (1990), a technology $C^{*}\left(q_{a}, q_{b}\right)$ such that $\hat{C}_{111}(q) \leq 0$ is said to be prudent. Kimball's concern was with how uncertainty and risk preferences affect savings behavior. Abstracting from the context, our problem possesses a formal similarity. Whereas Kimball studied how preference structures affect the deferral of (expected) consumption, we study how technology affects the deferral of production. In the face of higher storage costs, it is prudent to decrease the intensity of production whenever $\hat{C}_{111}(q) \leq 0 \forall q \geq 0$. This is because, for such a cost technology, seasonality in production keeps the marginal cost of production down while we know from Result 2(b) that higher storage costs reduce seasonal production behavior.

As for part $(b)$, we know from Result 2(c) that production decreases with an increase in nonseasonal costs. If $\hat{C}(q)$ is more convex at low output than at high output, then the incentive to vary production by season falls with an increase in parameter $m$. To bring the result toward a practical illustration, suppose that the cost of permanently employed labor increased. Then, for $\hat{C}_{111}(q) \leq 0 \forall q \geq 0$, both the equilibrium level of production and equilibrium production seasonality would fall. The effect on seasonality would provide a modicum of compensation for processors seeking a more stable supply of milk. Suppose it were true that large-scale and small-scale producers differ only by the draw from a mass distribution function of marginal cost $m, F(m): \overline{\mathbb{R}}_{+} \rightarrow \overline{\mathbb{R}}_{+}$with $d F(m) / d m \geq 0$. Then, for costs of form $C^{*}(q)$, an empirical observation that $\hat{q}$ and $\phi$ are negatively correlated across production units would provide evidence to support the hypothesis that $\hat{C}_{111}(q) \geq 0 \forall q \geq 0$. The value of stabilized production would be larger for larger producers, say with draw $m_{l}$, than for smaller producers with draw $m_{s}, m_{s}>m_{l}{ }^{16}$

Part $(c)$ is due to the fact that $\hat{q}$ increases with a decline in storage costs under the condition $\hat{C}_{111}(q) \leq 0 \forall q \geq 0$. When impediments to interseasonal transfers of production fall, the $\mathrm{n}$ it seems natural that more of the larger overall level of output will be consumed in season B. Under condition $\hat{C}_{111}(q) \geq 0 \forall q \geq 0$, the overall level of production decreases with a decline in $s$. More of what remains is allocated to season $\mathrm{B}$, so that the trough price must increase. 
Part $(d)$ is a consequence of part ( $a$ ). Part ( $a$ ) identifies conditions under which an increase in cost seasonality increases the overall production level. The change in production is distributed over seasons and has the same qualitative impact on both season price levels. Notice, though, that while $s$ and $-\rho$ have the same qualitative impacts on $\hat{q}$, they do not necessarily have the same qualitative impacts on season prices. This is because $s$ has a dual role in guiding production and consumption while the role of $\rho$ is focused on guiding production.

Precise conditions can be placed on the cost function such that mean production is invariant to the storage subsidy. Specifically, suppose that ${ }^{17}$

$$
\begin{gathered}
C(\hat{q}+\phi, \hat{q}-\phi)=G(\hat{q})+H(\phi)=G\left(\frac{1}{2} q_{a}+\frac{1}{2} q_{b}\right)+H\left(\frac{1}{2} q_{a}-\frac{1}{2} q_{b}\right), \\
G_{I}(\hat{q}) \geq 0, G_{11}(\hat{q})>0 \forall \hat{q} \geq 0 ; H(0)=0, H(\phi)=H(-\phi), H_{11}(\phi) \geq 0 \forall \phi \geq 0 .
\end{gathered}
$$

Then the following is readily established.

RESUlT 4. Under assumptions 1 and $2^{\prime}$, let $C\left(q_{a}, q_{b}\right)$ be of form (6). Then

a. $\quad d \hat{q} / d s=0$. That is, the level of production, $\hat{q}$, is invariant to a storage subsidy or a change in cost seasonality.

b. $\quad d \phi d d m=0$. That is, the level of production seasonality, $\phi$, is invariant to a change in nonseasonal costs.

These conclusions convey that an additively separable cost function, as given in specification (6), permits the moments of the production path to respond differently. The production index is insensitive to seasonality in costs, while the production seasonality index is insensitive to nonseasonal costs. ${ }^{18}$

It is interesting to compare Result 3 with Result 4 . Because the costs of changing the moments of production activities were not separated in cost specification $C^{*}(q)$, changes in the cost environment that should have a direct impact on a particular moment, either average level or the extent of seasonality, had an indirect impact on the other moment. This indirect effect does not occur in Result $4 .{ }^{19}$ 
To summarize this subsection, we have shown that the extent of temporal integration matters when seeking to understand how the economic and policy environment affects equilibrium. In our model, and regardless of technology asymmetries, price seasonality would disappear if storage costs were zero. Price seasonality would also disappear, regardless of storage costs, if there were no technology asymmetries. The nature of the technology also matters in more subtle ways. The cost function may express increasing, neutral, or decreasing technological preferences for seasonality as overall output increases. Results 3 and 4 present implications of these structural attributes for equilibrium.

\subsubsection{Quota}

Milk marketing quotas have been in place in the European Union since 1984. In this section we modify our more general model to accommodate this policy. We do so by fixing the level of production, $\hat{q}$, in welfare measure (1). In addition we make the following assumption.

ASSUMPTION 3. Markets for production rights are efficient, and quota is binding.

Then the optimality conditions are

(a) $\delta: P(\hat{q}-\delta)-P(\hat{q}+\delta)-s=0$,

(b) $\phi:-s-C_{I}(\hat{q}+\phi, \hat{q}-\phi)+C_{2}(\hat{q}+\phi, \hat{q}-\phi)+\rho=0$.

Dispensing with our linear demand assumption, we may present an omnibus result.

RESULT 5. Make assumptions 1, 2, and 3. Then:

a. $\quad d \delta / d s \geq 0 \geq d P(\hat{q}+\delta) / d s, d P(\hat{q}-\delta) / d s \geq 0$, and $d \phi / d s \leq 0$. That is, a storage subsidy decreases consumption seasonality and increases production seasonality. Consequently, peak price decreases and trough price increases with a storage subsidy.

b. $\quad d \delta / d m=d \phi / d m=0$. That is, a nonseasonal change in marginal costs has no effect on either consumption seasonality or production seasonality.

c. $\quad d \phi / d \rho \geq 0=d \delta / d \rho$. That is, an increase in cost seasonality increases production seasonality but has no effect on consumption seasonality. 
d. $\quad d \delta / d \hat{q} \geq(\leq) 0$ if $P_{11}(q) \geq(\leq) 0 \forall q \in \overline{\mathbb{R}}_{+}$, while $d P(\hat{q}-\delta) / d \hat{q} \leq 0$ and $d P(\hat{q}+\delta) / d \hat{q} \leq 0$ regardless. That is, an increase in the level of quota increases (decreases) consumption seasonality if the inverse demand function is convex (concave). Further, both peak and trough prices fall with an increase in the level of quota.

e. let $C\left(q_{a}, q_{b}\right)=C^{*}\left(q_{a}, q_{b}\right)$ as in Result 3. Then $d \phi / d \hat{q} \geq(\leq) 0$ if $\hat{C}_{111}(q) \leq(\geq) \quad 0 \forall q>0$. That is, production seasonality increases (decreases) with an increase in the level of quota if marginal cost is concave (convex).

f. let $C\left(q_{a}, q_{b}\right)$ assume the form given in equation (6). Then $d \phi / d \hat{q}=0$. That is, production seasonality is invariant to the level of quota.

Parts (a) through (c) are obvious, so we will not dwell upon them except to relate that any increase in nonseasonal costs would be absorbed in quota shadow rents. As for part $(d)$, if production increases, then prices fall in both seasons. If the inverse demand function is convex, then price becomes less sensitive to quantity variability. To support interseasonal arbitrage, a larger spread in consumption patterns must emerge. Part (e) may be motivated in a manner similar to Result $3(b)$, except more directly. Production level and production seasonality are complements in production when $\hat{C}_{111}(q) \leq 0 \forall q>0$. In the case of Result $5(f)$, the moments of production are, by design, additively separable.

Recapitulating this study of storage subsidies in a quota-regulated regime, as with the absence of quota restrictions it continues to be true that such subsidies act to decrease consumption seasonality and increase production seasonality. The effect of the aggregate quota level on consumption seasonality depends, however, on the curvature of the inverse demand function. This is because of the way in which storage costs and the demand function interact in distributing consumption across seasons. The effect of quota level on production seasonality is determined by whether the cost penalty for variability increases or decreases with an increase in overall output. A comparison of Result 5 with the findings in subsection 3.2.1. shows that the linear demand assumption is not trivial. Linear demand plays the same role on the consumption side as cost specification (6) does 
on the production side. It requires that the level of consumption has no effect on seasonality in consumption. In subsection 3.2.2, we can accommodate a more general demand environment because the production environment is simplified when quota fixes the overall level of production.

\subsection{Product Market Intervention}

Here, the regulator takes product off the market when the product price is low, i.e., in season A. In contrast to storage market intervention, the context of a product market intervention policy needs further clarification before engaging in an analysis. Specifically, it is necessary to assert what happens to the removed product. Either it is placed back on the market later or it does not reappear on the market. In the latter case, it might be placed on the animal feed market, as is often the case with SMP. Or subsidies may be paid, by the taxpayer at large, in order to place the product on world food markets.

Alternatively, the removed product might be destroyed.

If removed product is placed back on the market in season B, then, at least in our model, private storage will be crowded out one-for-one until public storage exceeds optimal storage and arbitrage constraint 2(a) no longer holds. Up to the point where there is complete crowding out, however, the production patterns of interest to this research are not affected. If the product is permanently removed from its primary market, then there will be implications for the level and seasonality of both equilibrium production and equilibrium price. Because permanent removal has more significant economic implications than does temporary removal, in the analysis to follow we consider only product market interventions under which the product is not placed back in the same market later.

\subsubsection{No Quota}

The policy takes the form of product removal of amount $\kappa$ in surplus season A. This product is destroyed immediately after removal. So $\hat{q}+\phi$ is produced in that season and $\hat{q}+\delta$ is consumed in that season. Surplus production amounts to $\phi-\delta$. Upon removing $\kappa$, the amount $\phi-\delta-\kappa$ is stored for consumption in season B. Adding stored production to season B production, $\hat{q}-\phi$, we have that $\hat{q}-\delta-\kappa$ is placed on the market in season B. In season A, $\hat{q}+\delta+\kappa$ disappears from the market 
through consumption or (paid) removal. Consequently, season A industry receipts are $P(\hat{q}+\delta)(\hat{q}+\delta+\kappa)$ where price must be consistent with consumption $\hat{q}+\delta$.

Season B industry receipts are $P(\hat{q}-\delta-\kappa)(\hat{q}-\delta-\kappa)$. Aggregate industry welfare, summed over producers and processors, is

$$
\begin{gathered}
\Pi[\delta, \phi, \hat{q}, \kappa]=P(\hat{q}-\delta-\kappa)(\hat{q}-\delta-\kappa) \\
+P(\hat{q}+\delta)(\hat{q}+\delta+\kappa)-(\phi-\delta-\kappa) s-C(\hat{q}+\phi, \hat{q}-\phi)-m \hat{q}+\rho \phi .
\end{gathered}
$$

We see that mean consumption over the two periods is $\hat{q}-0.5 \kappa$, with consumption deviations of the form $\delta+0.5 \kappa$ and $-\delta-0.5 \kappa$ so that the amplitude of the consumption pattern is $2 \delta+\kappa$. The cost to the policymaker of the product removal policy is $P(\hat{q}+\delta) \kappa$. Product destruction costs have been assumed to be negligible.

Optimization over the three choice variables, and making the linear demand assumption, identifies the equilibrium conditions

(a) $\delta: 2 \delta+\kappa=s / \alpha_{1}$,

(b) $\phi:-C_{1}(\hat{q}+\phi, \hat{q}-\phi)+C_{2}(\hat{q}+\phi, \hat{q}-\phi)=s-\rho$,

(c) $\hat{q}: 2 \alpha_{0}-2 \alpha_{1} \hat{q}+\alpha_{1} \kappa-C_{1}(\hat{q}+\phi, \hat{q}-\phi)-C_{2}(\hat{q}+\phi, \hat{q}-\phi)=m$.

Notice that the amplitude of consumption is given on the left-hand side of ( $8 a)$. A study of this system provides the following.

RESULT 6. Make assumptions 1 and 2 ' under a product removal policy. Then:

a. $\quad d(2 \delta+\kappa) / d \kappa=0, d \hat{q} / d \kappa \geq 0, d P(\hat{q}-\delta) / d \kappa \geq 0$, and $d P(\hat{q}+\delta) / d \kappa \geq 0$. That is, product removal has no effect on consumption seasonality, increases production, and increases price in both seasons.

b. let $C\left(q_{a}, q_{b}\right)=C^{*}\left(q_{a}, q_{b}\right)$ as in Result 3. Then $d \phi / d \kappa \geq(\leq) 0$ if $\hat{C}_{111}(q) \leq(\geq) \quad 0 \forall q>0$. That is, production seasonality increases (decreases) with an increase in the level of product removal if marginal cost is concave (convex).

c. let $C\left(q_{a}, q_{b}\right)$ assume the form given in equation (6). Then $d \phi / d \kappa=0$. That is, production seasonality is invariant to the level of product removal. 
The opportunity to smooth consumption that is afforded by storage infrastructure takes much of the bite out of a policy that would seem to be targeted at altering seasonal behavior. Indeed, the policy does not affect consumption seasonality or price seasonality when demand is linear. And, as some additional algebra would show, the price impacts due to nonlinear inverse demand could increase or decrease price seasonality. Predictably, overall production is elevated by the intervention policy because the storage link requires that all prices rise together. Thus, assuming that storage markets function adequately, the seasonspecific intervention policy should be seen as a pure price support mechanism rather than as an attempt to redress any seasonality related "disturbances" or disequilibria.

Concerning parts $(b)$ and $(c)$, at first blush it might appear that removals targeted in the high production season would only exacerbate production seasonality. Again, however, market integration through storage requires that production incentives increase in both seasons, and any bias toward the low-cost season would have little to do with pure price incentives. Rather, the bias would emerge largely as a consequence of the nature of the underlying production technology.

To review the effects of production removal under linear demand, the overall price level is supported and the level of consumption seasonality is not affected by the policy. Production seasonality may rise or fall, but intertemporal arbitrage through storage ensures that consumption seasonality is not affected.

\subsubsection{Quota}

As in subsection 3.2.2., in this section we fix the level of production, $\hat{q}$. But we do not impose Assumption 2', so that system (8) becomes

(a) $\delta: P\left(q^{*}-\delta^{*}\right)-P\left(q^{*}+\delta^{*}\right)=s$,

(b) $\phi:-C_{1}(\hat{q}+\phi, \hat{q}-\phi)+C_{2}(\hat{q}+\phi, \hat{q}-\phi)=s-\rho$,

where $q^{*}=\hat{q}-0.5 \kappa$ and $\delta^{*}=\delta+0.5 \kappa$. A study of this system establishes the following.

RESULT 7. Make assumptions 1, 2, and 3 under a product removal policy. Then $d \delta^{*} / d \kappa \leq(\geq) 0$ if $P_{11}(q) \geq(\leq) 0 \forall q \in \overline{\mathbb{R}}_{+}$, and $d \phi / d \kappa=0$ regardless. That is, an 
increase in the level of removed product decreases (increases) consumption seasonality if the inverse demand function is convex (concave). It has no effect on production seasonality.

The effects of altered production quota are the same as in Result 5 and warrant no further discussion. When compared with a storage subsidy, observe that product removal is a more targeted policy instrument in that it does not alter production patterns under a quota regime. Absent production control, the effect of product removal on production seasonality was only as a side effect of alternating the incentive to change overall production. Whether the greater focus of the instrument is good or bad depends on what the policymaker seeks to achieve, and we are not in a position to suggest the better instrument.

\section{Discussion}

Seasonality in production is one of the peculiarities of many agricultural activities. Its consequences at the market level are a concern for producers, processors, and policymakers. The consequences may also be a concern for consumers, particularly consumers in countries where transportation and storage infrastructure do not function well. ${ }^{20}$ Yet little has been written on the production economics of seasonality.

Our goal in this paper has been to understand the determinants of endogenous seasonal production. From a policy perspective, this is an issue in animal agriculture as it applies to Northwest Europe. Yet, and perhaps partly because the pathways of endogenous seasonal production are not well understood, there would seem to be confusion about how policy might affect storage as well as seasonal aspects of production, consumption, and price.

Using tools appropriate for accommodating asymmetries in seasonal production environments, we have developed a simple yet versatile market level model of seasonal production when product can be stored. It quickly becomes obvious, when applying the model to study a subsidy on the private storage of milk products, that dampened price seasonality is traded off against amplified production seasonality. In the absence of quota limits, production may rise or fall with the storage subsidy. Product market removals support the level of product price and the incentive to produce. Determinate effects on these seasonality indices can be identified if we are prepared to speculate about the true nature of demand and cost technologies. Because we have found that plausible assump- 
tions on the production technology and the market-level demand can support a variety of market-level production patterns, empirical studies will be required if implications are to be drawn for policy formation.

Our analysis suggests that E.U. policy on agricultural seasonality has not been clearly thought through. Are season-varying policy interventions and aid for private storage really intended solely as income support policies? If not, what are the negative externalities associated with seasonality? Are they most directly associated with quantity seasonalities or with price seasonality? Seasonal production may have adverse food safety implications, but aids to private storage will only exacerbate the problem, and product market intervention might also add to the harm. Seasonal consumption might have implications for balance in diets among citizens of E.U. countries. But consumption-side pressure groups do not appear to motivate the policies. And what, specifically, might the nature of any consumption-side externalities be?

Returning to the empirical evidence on seasonal trends in milk production, but away from policy issues, several conjectures warrant formal and empirical investigation. It would seem that dairy innovations in feed sources, in housing, and in reproduction technologies have possessed a bias toward reducing seasonality. How might any seasonal biases in innovations be measured, and are the incentives in place for commercial companies to research and develop seasonality-reducing technologies? Might distance from the market be a factor in Ireland's persistent quantity seasonality? The Netherlands is also a significant dairy product exporter, and yet production seasonality is not as strong there. In 2000, its standard deviation of shares in monthly production was 0.037 , a number that is somewhat less than that of Ireland (0.044) but substantially larger than the standard deviations in the United Kingdom (0.006) and France (0.009). The dairy product profile produced in the Netherlands is more market oriented, with stronger emphases on cream and whole milk products rather than storable butter and milk powders. Dutch production practices are also quite different from those in Ireland. It seems natural to ask whether cost seasonality might interact with the incentives to add value in determining the amount and variety of dairy processing. More broadly, are there multiple possible equilibria such that a concerted effort, at a national or processor level, might achieve a superior selfsustaining pattern of industry activities? 


\section{Endnotes}

1. We have pointed to several problems that arise from seasonality. But, of course, we are not suggesting that all give rise to suboptimal welfare given the available technology set. Food safety issues, when combined with imperfect information, will likely generate negative externalities. Then there may be a role for policy interventions that seek to deseasonalize production. The cost technologies that underlie seasonal production do not, however, in themselves constitute externalities. This distinction would not appear to be recognized in most discussions on policy interventions to alter seasonal patterns in prices, production, and consumption.

2. Research by, e.g., Crosse, O'Brien, and Ryan (2000), and Ryan, Crosse, and Fitzgerald (2000) highlight the feasibility of autumn-calving herd management in Ireland and suggest possible adjustments to alter production.

3. See Allen and Lueck 1998 for interesting insights on trends toward nonseasonal production in animal agriculture. In the case of dairying, Caine and Stonehouse (1983) refer to studies that identify a deseasonalizing bias in progressive husbandry practices. An argument could be made that artificial insemination promotes seasonality by allowing the producer to concentrate calving activities in a small time interval so that dedicated staff and resources can be made available. At the market level, any such endeavors to concentrate calving will likely be lost as different firms spread calving over time so that any economic rents from a particular choice of calving period are exhausted.

4. Milk production seasonality is also an issue in the United States. Washington, Lawson, and Kilmer (2000) document that Florida dairy cooperatives have faced a supplier production peak/trough ratio of 1.35 to 1.40 in the early 1990 s. These cooperatives have sought to reduce production seasonality by implementing a voluntary seasonal pricing schedule.

5. For a review of the CAP, see European Commission 1997, 1999; or Agra Europe 2002.

6. The European Union also operates a deseasonalization premium for beef slaughter. If autumn slaughter of male bovines exceeds 35 percent of annual slaughter, then a premium of between 18 and 73 Euro per head is paid per male bovine slaughtered during the spring. 
7. There is a large body of insightful work on agricultural production that might be labeled as two-season models, e.g., Innes 1993 and Saha 1994. These generally deal with crop production, where there is a growing season and a nongrowing season. Naturally, the issue of endogenous production seasonality is precluded by construction.

8. The aggregate cost function must be consistent with the firm-level cost functions, and it is unlikely to be a linear sum of firm-level cost functions. See, e.g., Chambers 1988 on cost function aggregation.

9. Most costs have a seasonal component. To distinguish between seasonal and nonseasonal components of some function $g\left(q_{1}, q_{2}\right)$, one might remove the largest symmetric convex function among the set of symmetric and convex functions $h\left(q_{1}, q_{2}\right) \in S C$, i.e., $h^{*}\left(q_{1}, q_{2}\right)=\max \left\{h\left(q_{1}, q_{2}\right): g\left(q_{1}, q_{2}\right) \geq h\left(q_{1}, q_{2}\right), h\left(q_{1}, q_{2}\right) \in S C\right\}$. Residual $g\left(q_{1}, q_{2}\right)-h^{*}\left(q_{1}, q_{2}\right)$ might be defined as the seasonal component of costs.

10. Turkeys and Easter lamb are the obvious exceptions. In any case, our model can be readily adapted to study seasonality that originates on the demand side.

11. Linearity of storage costs is not a trivial assumption. As will be seen later, the assumption allows a measure of separation between production and consumption.

12. Higher-order derivatives are represented in the obvious way. For example, we will denote by $h_{i j}(\cdot)$ the second derivative of $h(\cdot)$ with respect to the $i$ th and $j$ th arguments.

13. "Permutation symmetric" means that the function value is invariant under any interchange in the value of a pair of arguments. For example, $g\left(z_{1}, z_{2}, z_{3}\right)=g\left(z_{2}, z_{1}, z_{3}\right)$ for any admissible value of the triple. Also, $g(\vec{z})$ need not be convex for relation (3) to hold. Given symmetry, the less restrictive condition of "Schur-convex" suffices.

14. One may wonder why symmetry is assumed when Result 1 (and also other results to follow) may seem so intuitive. Indeed, were it assumed that $C_{12}=0 \forall\left(q_{a}, q_{b}\right)$ then more conventional conditions on marginal cost functions would immediately yield the finding. However, when $C_{12} \neq 0$, then a change in either argument affects both marginal cost functions, and intuition developed for unidimensional functions does not apply.

15. See Silberberg 1978 (p. 290) on reciprocity.

16. We consider $\phi$, and not $\phi / \hat{q}$, to be the measure of seasonality. So the coefficient of variation would be even more stable as the draw on $m$ decreases and the scale of production increases. 
17. The functional form in (6) is d'Alambert's representation of the general solution to the wave equation. See p. 658 of Kreyszig 1988. The multiplier $1 / 2$ is to introduce into functions $G(\cdot)$ and $H(\cdot)$ in order to convert the arguments into a mean and deviation format.

18. One might view specification (6) as the cost seasonality analog to the Just and Pope (1978) specification of stochastic primal production technologies.

19. Part (a) of Result 3 suggests that cost functions that satisfy $\hat{C}_{111}(q)=0 \forall q \geq 0$ should have special significance. This is the set of quadratic cost functions, and an appeal to continuity would suggest the intuition that the conclusions of Result 4 should also pertain for cost functions of the form $\hat{C}(q)=\tau_{0}+\tau_{1} q+\tau_{2} q^{2}$. In fact, quadratic cost functions comprise a special case of specification (6). To illustrate, set $\tau_{0}=\tau_{1}=0$ with $\tau_{2}=1$. Then write $q_{a}^{2}+q_{b}^{2} \equiv\left(q_{a}+q_{b}\right)^{2}-2 q_{a} q_{b} \equiv 4 \hat{q}^{2}$ $-2(\hat{q}+\phi)(\hat{q}-\phi) \equiv 2 \hat{q}^{2}+2 \phi^{2}$ so that $G(\hat{q})=2 \hat{q}^{2}, H(\phi)=2 \phi^{2}$, and all the conditions in specification (6) are satisfied.

20. One school of thought in Economic History holds that the nature of production seasonality in agriculture can be an important determinant of the path toward economic development (Sokoloff and Dollar, 1997). 


\section{Appendix}

Proof of Result 1. From relation (4), if $\rho>s$ then $\phi \geq 0$. From equation (2b), $\rho>s$ implies that $C_{1}(\hat{q}+\phi, \hat{q}-\phi)>C_{2}(\hat{q}+\phi, \hat{q}-\phi)$. Given symmetry and strict convexity, this is inconsistent with $\phi=0$. To see the inconsistency, write $L(\phi)=$ $C_{1}(\hat{q}+\phi, \hat{q}-\phi)-C_{2}(\hat{q}+\phi, \hat{q}-\phi)$ so that $L(0)=0$ and $L_{1}(\phi)=C_{11}+C_{22}-2 C_{12}>0$. The latter inequality is formally demonstrated in Result 2 . Therefore, $\phi>0$.

Now suppose that $\phi<\delta$ so that the cost of storage in equation (1) is $(\delta-\phi) s$. We will prove that this generates a contradiction for $s$ sufficiently small that an interior storage solution exists. In particular, system (2) would be written as

(a) $\delta: P(\hat{q}-\delta)-P(\hat{q}+\delta)+s=0$,

(b) $\phi: s-C_{1}(\hat{q}+\phi, \hat{q}-\phi)+C_{2}(\hat{q}+\phi, \hat{q}-\phi)+\rho=0$,

(c) $\hat{q}: P(\hat{q}-\delta)+P(\hat{q}+\delta)-C_{1}(\hat{q}+\phi, \hat{q}-\phi)-C_{2}(\hat{q}+\phi, \hat{q}-\phi)-m=0$,

so that (A1a) implies $\delta<0$. But $\rho+s>0$ so that (A1b) gives $C_{1}(\hat{q}+\phi, \hat{q}-\phi)$ $>C_{2}(\hat{q}+\phi, \hat{q}-\phi)$, while the Ostrowski condition, symmetry, and strict convexity imply that $\phi>0$. Hence, $\phi>\delta$, and a contradiction has been generated. Therefore, the only consistent case is where $\phi \geq \delta$. So (1) is correctly posed. And (2a), together with strict monotonicity of $P(q)$, then implies that $\delta>0$.

Proof of Result 2. Part (a) is immediate from equation $\left(2^{\prime} a^{\prime}\right)$. For part (b), differentiate equations $\left(2^{\prime} b^{\prime}\right)-\left(2^{\prime} c^{\prime}\right)$ with respect to vector $(\hat{q}, \phi, s)$ to obtain

$$
\left(\begin{array}{rr}
C_{22}-C_{11} & 2 C_{12}-C_{11}-C_{22} \\
-\left(2 \alpha_{1}+C_{11}+2 C_{12}+C_{22}\right) & C_{22}-C_{11}
\end{array}\right)\left(\begin{array}{l}
d \hat{q} / d s \\
d \phi / d s
\end{array}\right)=\left(\begin{array}{l}
1 \\
0
\end{array}\right) .
$$


Inversion yields

$$
\left(\begin{array}{l}
d \hat{q} / d s \\
d \phi / d s
\end{array}\right)=\frac{1}{M}\left(\begin{array}{c}
C_{22}-C_{11} \\
2 \alpha_{1}+C_{11}+2 C_{12}+C_{22}
\end{array}\right)
$$

where $M=4 C_{12}^{2}-4 C_{11} C_{22}+2 \alpha_{1}\left(2 C_{12}-C_{11}-C_{22}\right)$. Cost function convexity ensures that $4 C_{12}^{2}-4 C_{11} C_{22}<0$. Convexity also ensures that, regardless of the sign of $C_{12}$, the inequality $\left|C_{12}\right|\left\langle\left(C_{11} C_{22}\right)^{0.5}\right.$ adheres. Thus, $\left.C_{11}+C_{22}=2\left(C_{11} C_{22}\right)^{0.5}+\left(C_{11}^{0.5}-C_{22}^{0.5}\right)^{2}\right\rangle$ $2\left|C_{12}\right|+\left(C_{11}^{0.5}-C_{22}^{0.5}\right)^{2} \geq 2\left|C_{12}\right|$. Therefore $C_{11}+C_{22}>2 C_{12}$ and $C_{11}+C_{22}>-2 C_{12}$. Since $\alpha_{1}>0$, it follows that $M<0$ and that $d \phi / d s \leq 0$. As for the remainder of part $(b)$, a differentiation of $\left(2^{\prime} b^{\prime}\right)-\left(2^{\prime} c^{\prime}\right)$ with respect to vector $(\hat{q}, \phi,-\rho)$ generates equation (A3) except that $-\rho$ replaces $s$.

Regarding part $(c)$, differentiate equations $\left(2^{\prime} b^{\prime}\right)-\left(2^{\prime} c^{\prime}\right)$ with respect to vector $(\hat{q}, \phi, m)$ to generate

$$
\left(\begin{array}{rr}
C_{22}-C_{11} & 2 C_{12}-C_{11}-C_{22} \\
-\left(2 \alpha_{1}+C_{11}+2 C_{12}+C_{22}\right) & C_{22}-C_{11}
\end{array}\right)\left(\begin{array}{l}
d \hat{q} / d m \\
d \phi / d m
\end{array}\right)=\left(\begin{array}{l}
0 \\
\\
1
\end{array}\right)
$$

with inverse

$$
\left(\begin{array}{l}
d \hat{q} / d m \\
d \phi / d m
\end{array}\right)=\frac{1}{M}\left(\begin{array}{c}
C_{11}-2 C_{12}+C_{22} \\
C_{22}-C_{11}
\end{array}\right)
$$

Part (c) then follows. To verify part (d), use (A3) and (A5) to observe that $d \hat{q} / d s=-d \hat{q} / d \rho=\left(C_{22}-C_{11}\right) / M=d \phi / d m$.

Proof of Result 3. From (A3) we have that $d \hat{q} / d s=\left[\hat{C}_{11}(\hat{q}-\phi)-\hat{C}_{11}(\hat{q}+\phi)\right] / M$.

From Result 1 we know that $\phi>0$. If $\hat{C}_{11}(q)$ is an increasing function, then 
$\hat{C}_{11}(\hat{q}-\phi)<\hat{C}_{11}(\hat{q}+\phi)$ and $d \hat{q} / d s>0$. A change in $-\rho$ acts in like fashion. Part $(b)$ follows from a similar analysis of (A5).

For part $(c)$, we use the lock separability of system $\left(2^{\prime}\right)$ to arrive at $d(\hat{q}+\delta) / d s=0.5 \alpha_{1}^{-1}+\left[\hat{C}_{11}(\hat{q}-\phi)-\hat{C}_{11}(\hat{q}+\phi)\right] / M>0$ if $\hat{C}_{111}(q) \geq 0 \forall q>0$, and $d(\hat{q}-\delta) / d s=-0.5 \alpha_{1}^{-1}+\left[\hat{C}_{11}(\hat{q}-\phi)-\hat{C}_{11}(\hat{q}+\phi)\right] / M<0$ if $\hat{C}_{111}(q) \leq 0 \forall q>0$. The relationships for price follow immediately. Upon observing that $\rho$ alters $\hat{q}$ but does not affect $\delta$, then it can be seen that part $(d)$ follows from part $(a)$.

Proof of Result 4. In each case, $C_{11}=C_{22}$. Then apply (A3) and (A5).

Proof of Result 5. Part (a) is immediate from $\left(2^{\prime \prime} a\right),\left(2^{\prime \prime} b\right)$, and the fact that $C_{11}+C_{22}>2 C_{12}$, as established in Result 2. Part $(b)$ is also immediate. In $(c)$, an increase in cost seasonality has no effect on $\left(2^{\prime \prime} a\right)$, and it act like a storage subsidy in $\left(2^{\prime \prime} b\right)$.

For part $(d)$, note that storage costs operate as a separation parameter in system $\left(2^{\prime \prime}\right)$, so that we can shock each equation without regard to spillovers. We have $d \delta / d \hat{q}=$ $\left[P_{1}(\hat{q}-\delta)-P_{1}(\hat{q}+\delta)\right] /\left[P_{1}(\hat{q}-\delta)+P_{1}(\hat{q}+\delta)\right]$ where the denominator is negative. The numerator is negative (positive) when inverse demand is convex (concave). For parts $(e)$ and $(f)$, differentiate $\left(2^{\prime \prime} b\right)$ and then apply analysis in Results 3 and 4.

Proof of Result 6. Note that $(8 b)$ and $(8 c)$ determine $\hat{q}$ and $\phi$. Equation $(8 a)$ is block separated in determining the equilibrium value of $2 \delta+\kappa$. Indeed, the equilibrium value of $2 \delta+\kappa$ is invariant to $\kappa$ since $s / \alpha_{1}$ is invariant to $\kappa$. And so the value of $\kappa$ does not affect consumption seasonality.

Differentiating $(8 b)-(8 c)$ with respect to the vector $(\hat{q}, \phi, \kappa)$ provides

$$
\left(\begin{array}{rr}
C_{22}-C_{11} & 2 C_{12}-C_{11}-C_{22} \\
2 \alpha_{1}+C_{11}+2 C_{12}+C_{22} & C_{11}-C_{22}
\end{array}\right)\left(\begin{array}{l}
d \hat{q} / d \kappa \\
d \phi / d \kappa
\end{array}\right)=\left(\begin{array}{l}
0 \\
\alpha_{1}
\end{array}\right)
$$

Inversion yields 


$$
\left(\begin{array}{c}
d \hat{q} / d \kappa \\
d \phi / d \kappa
\end{array}\right)=-\frac{\alpha_{1}}{M}\left(\begin{array}{c}
C_{11}-2 C_{12}+C_{22} \\
C_{22}-C_{11}
\end{array}\right)
$$

where the expression for $M$ is as in the proof of Result 2. It follows that $d \hat{q} / d \kappa \geq 0$ from (A7), convexity of $C\left(q_{a}, q_{b}\right)$, and the inequality $C_{11}+C_{22} \geq 2 C_{12}$ demonstrated in Result 2. As for prices, employ ( $8 a$ ) to write $\hat{q}-\delta-\kappa$ as $\hat{q}-0.5 \kappa-0.5 s / \alpha_{1}$ so that $d(\hat{q}-\delta-\kappa) / d \kappa=d \hat{q} / d \kappa-0.5$. System (A7) then yields $d(\hat{q}-\delta-\kappa) / d \kappa=$ $2\left(C_{11} C_{22}-C_{12}^{2}\right) / M<0$ so that intervention increases price in the peak price period. Similarly, $(8 a)$ establishes that $d(\hat{q}+\delta) / d \kappa=d \hat{q} / d \kappa-0.5<0$ so that intervention also increases price in the trough price period.

For parts $(b)$ and (c), refer to Result 3 and Result 4 when studying (A7).

Proof of Result 7. For production seasonality, note that $\kappa$ does not enter equation $\left(8^{\prime} b\right)$. This reflects the fact that, under a quota regime, the firms seek only to minimize costs and do not care whether the product is destined for a landfill or consumption. As to consumption seasonality, please see Result $5(d)$. The effect documented there also describes the effect of product removal because storage allows the consequences of product removal to be smoothed over both seasons. 


\section{References}

Agra Europe. 2002. CAP Monitor. London, UK: Cambridge University Press.

Allen, D.W., and D. Lueck. 1998. "The Nature of the Farm.” Journal of Law and Economics 41(2, October): 343-86.

Bell, R.G. 1997. "Distribution and Sources of Microbial Contamination on Beef Carcasses." Journal of Applied Microbiology 82: 292-300.

Caine, R.J.H., and D.P. Stonehouse. 1983. "Adjusting the Seasonality of Milk Shipments in Canada: Problems, Economic Impacts and Potential Policies." Canadian Journal of Agricultural Economics 31(November): 331-50.

Chambers, R.G. 1988. Applied Production Analysis: A Dual Approach. Cambridge, UK: Cambridge University Press.

Chambers, R.G., and J. Quiggin. 1997. "Separation and Hedging Results with State-Contingent Production." Economica 64(254, May): 187-209.

-2000. Uncertainty, Production, Choice, and Agency: The State-Contingent Approach. Cambridge, UK: Cambridge University Press.

Crosse, S., B. O'Brien, and G. Ryan. 2000. "Milk Production and Seasonality-Recommended Moorepark Systems for a Diversifying Food Industry.” Moorepark Working Paper, Teagasc, Cork, Ireland.

European Commission. 1997. "Situation and Outlook: Dairy Sector." Document SEC(97) 1013. Directorate General for Agriculture, Brussels, Belgium. April.

1999. “The CAP Reform: Milk and Milk Products. Factsheet.” Directorate General for Agriculture. Brussels, Belgium. November.

Innes, R. 1993. "Two-Season Subsistence Farming, Urban Food Subsidies and Optimal Agricultural Policy." Oxford Economic Papers 45(4, October): 668-90.

Just, R.E., and R.D. Pope. 1978. "Stochastic Specification of Production Functions and Economic Implications. Journal of Econometrics 7(1, February): 67-86.

Kimball, M.S. 1990. "Precautionary Saving in the Small and in the Large." Econometrica 58(January): 5373.

Kreyszig, E. 1988. Advanced Engineering Mathematics, 6th ed. New York: John Wiley \& Sons.

Marshall, A.W., and I. Olkin. 1979. Inequalities: Theory of Majorization and its Applications. New York: Academic Press.

Mas-Colell, A., M.D. Whinston, and G. Green. 1995. Microeconomic Theory. Oxford, UK: Oxford University Press. 
Ryan, G., S. Crosse, and S. Fitzgerald. 2000. "The Development of Feeding and Management Strategies for Milk Production Systems with Very Contrasting Milk Supply Patterns." Final Report, Project Number 4169, Dairy Levy Series No. 4. Teagasc, Dublin, Ireland.

Saha, A. 1994. "A Two-Season Agricultural Household Model of Output and Price Uncertainty." Journal of Development Economics 45(2, December): 245-69.

Sheridan, J.J. 1998. "Sources of Contamination During Slaughter and Measures for Control. Journal of Food Safety 18: 321-39.

Silberberg, E. 1978. The Structure of Economics: A Mathematical Analysis. New York: McGraw-Hill.

Sokoloff, K.L., and D. Dollar. 1997. "Agricultural Seasonality and the Origin of Manufacturing in Early Industrial Economies: The Contrast between England and the United States." Journal of Economic History 57(June): 288-321.

Washington, A.A., R.W. Lawson, and R.L. Kilmer. 2000. "An Evaluation of the Effectiveness of the Florida Cooperative's Seasonal Pricing Plan on Seasonal Production Variability.” Journal of Agricultural and Applied Economics 31(1, April): 113-21. 